\title{
Perfil das pessoas com lesão por pressão na reabilitação: relação entre braden e dependência funcional
}

\section{Profile of people with pressure injury in rehabilitation: relationship between braden and functional dependence}

\author{
Karla Pereira Cândido' • Juliana Caldas de Souza² • Fernanda Miranda de Oliveira ${ }^{3}$
}

\begin{abstract}
RESUMO
O objetivo deste estudo foi traçar o perfil das pessoas com Lesão por Pressão atendidas em um Centro Estadual de Reabilitação na cidade de Goiânia e, avaliar a associação entre as variáveis da escala de Medida de Independência Funcional e escala de Braden. Trata-se de um estudo transversal, descritivo, com abordagem quantitativa. Foram entrevistadas 54 pessoas, das quais houve predominância do sexo masculino, idade adulto-jovem, baixo nível de escolaridade e baixa renda familiar. Os diagnósticos mais relevantes, foram traumatismo raquimedular, seguido de acidente vascular cerebral, sendo tetraplegia a sequela mais dominante. De acordo com a escala de Medida de Independência Funcional houve maior ênfase na dependência total para o domínio motor, e conforme a escala de Braden a maioria apresentaram alto risco de desenvolver Lesão por Pressão, foi confirmado uma correlação positiva e moderada entre essas escalas, sendo que a dependência funcional pode ser um dos preditores do risco de desenvolver Lesão por Pressão. Podemos concluir que reconhecer características da população assistida favorece um melhor planejamento das ações preventivas e que ampliar as formas de diagnóstico precoce do risco de desenvolver Lesão por Pressão, contribui para o trabalho interdisciplinar, garantindo melhor qualidade de vida ao paciente.
\end{abstract}

Palavras chave: Lesão por Pressão. Doenças do Sistema Nervoso. Centros de Reabilitação

\begin{abstract}
The objective of this study was to trace the profile of people with pressure injuries treated at a State Rehabilitation Center in the city of Goiânia and to evaluate the association between the variables of the Functional Independence Measure and Braden scale. It is a cross-sectional, descriptive study with a quantitative approach. Fifty-four people were interviewed, of which there was a predominance of males, young adult age, low educational level and low family income. The most relevant diagnoses were spindle trauma, followed by stroke, with tetraplegia being the most dominant sequel. According to the Functional Independence Measure scale, there was greater emphasis on total motor domain dependence, and according to the Braden scale most were at high risk of developing Pro-Pressure Injury, a positive and moderate correlation was confirmed between these scales, being that functional dependence may be one of the predictors of the risk of developing Pro-Pressure Injury. We can conclude that recognizing characteristics of the assisted population favors a better planning of preventive actions and that expanding the early diagnosis of the risk of developing pressure injury contributes to the interdisciplinary work, guaranteeing a better quality of life for the patient.
\end{abstract}

Keywords: Pressure Injury. Nervous System Diseases. Rehabilitation Centers

'Enfermeira, Residente em Saúde Funcional e Reabilitação do Programa de Residência Multiprofissional da Secretaria do Estado de Saúde de Goiás. Endereço para correspondência: Rua Dona Mariquinha, qd.I0 lt.0I, Setor Negrão de Lima, Goiânia, Goiás. E-mail: contato.karlapereira@hotmail.com

²Enfermeira, Mestre em enfermagem e Tutora do Programa de Residência em Enfermagem no Centro Estadual de Reabilitação e Readaptação Dr. Henrique Santillo, Goiânia, Goiás. E-mail: julianacaldas8@gmail.com

${ }^{3}$ Mestre em enfermagem, Enfermeira no Centro Estadual de Reabilitação e Readaptação Dr. Henrique Santillo, Goiânia, Goiás. E-mail: fernanda0I03।988@hotmail.com 


\section{INTRODUÇÃO}

A Lesão por Pressão (LPP) ainda é uma das principais complicações em pacientes com sequelas neurológicas, mesmo sendo passível de ações preventivas. É capaz de desenvolver repercussões negativas físicas e emocionais de grande impacto na vida social do indivíduo, além de prejudicar o processo de reabilitação, aumentar tempo de permanência hospitalar e ainda potencializar o risco à infecção, aumentando a taxa de mortalidade ${ }^{(1)}$.

O Centro Especializado em Reabilitação (CER) é um ambiente hospitalar com atendimento direcionado para o grande incapacitado, sendo que a instituição em estudo oferta atendimentos exclusivamente via Sistema Único de Saúde (SUS), sendo reconhecida pelo Ministério da Saúde (MS) como CER IV, por atender pessoas com deficiência física, auditiva, visual e intelectual.

Dentre as lesões neurológicas que acometem o sistema nervoso central e periférico, ressaltamos algumas potencialmente incapacitantes por alterar diretamente a mobilidade física do indivíduo, como o Traumatismo Cranioencefálico (TCE) e Traumatismo Raquimedular (TRM), atualmente ocupam os primeiros lugares no ranking brasileiro de traumas, comumente resultantes de acidentes automobilísticos que afetam em sua maioria, pessoas jovens em boas condições de saúde ${ }^{(2)}$.

O grau da limitação física reflete diretamente no nível de incapacidade do indivíduo em realizar as atividades da vida diária, geralmente a mobilidade prejudicada dispõe maior risco de desenvolver LPP. Além de alterações vesicais e intestinais, caracterizado por bexiga e/ou intestino neurogênico, responsáveis pelo aumento da umidade em regiões perineais através das perdas involuntárias em fralda, que também favorecem o desenvolvimento de dermatites e lesões cutâneas ${ }^{(3)}$.

Para avaliação da dependência funcional podemos utilizar a escala de Medida de Independência Funcional (MIF), que avalia 18 afazeres básicos e essenciais à rotina diária, agrupados em tarefas de autocuidado, transferências, controle esfincterianos, locomoção e habilidades cognitivas, assim, cada tópico avaliado recebe uma pontuação que varia de um a sete, de acordo com o grau de ajuda que o indivíduo necessita para conclusão da atividade, sendo que um significa ajuda total e sete, a independência completa ${ }^{(3)}$.

Seu escore resultante pode ser classificado em dependência baixa, variando de 80 a 126, dependência moderada com escore de 37 a 79 e dependência completa com resultados de 18 a 36. Cada classe pode corresponder a necessidade de cuidados descrita em horas, sendo a baixa equivalente até duas horas; a moderada entre três e seis horas e a alta mais de sete horas de cuidados intermitentes ${ }^{(3-4)}$.

Destacando que LPP é uma das principais compli- cações ao paciente acamado ou que tenha mobilidade prejudicada e sendo esta, uma complicação passível de prevenção, o uso de instrumento ou escala para avaliação do risco, é imprescindível na detecção precoce de fatores predisponentes ${ }^{(5)}$.

Outra escala que pode ser utilizada é a escala de Braden, que permite uma avaliação integrativa dos fatores de risco para desenvolvimento de LPP, abrangendo alterações na mobilidade, atividade e percepção sensorial, nutrição, umidade e fricção e cisalhamento. Cada subescala pode ser pontuada de um a quatro, exceto a de fricção e cisalhamento que pode ser pontuada de um a três. A soma dos subescores, resultará em valores que podem variar de seis a vinte e três, é comumente dividida de acordo com o nível de exposição em risco baixo, referente a pontuação entre 15 e 18 , risco moderado que abrange escores entre 13 e 14 , risco alto indicado por pontuações entre 10 a 12 e risco altíssimo quando o resultado é menor que nove ${ }^{(5)}$.

Diante do exposto, surge uma questão norteadora: Mesmo sendo passível de ações preventivas, porque as lesões por pressão ainda são frequentes em ambiente hospitalar? Conhecer características sociodemográficas e ampliar a disponibilidade de escalas de avaliação contribuiria para o diagnóstico precoce?

Sendo assim, o objetivo do estudo foi identificar o perfil das pessoas com LPP, atendidos em um centro de reabilitação, proporcionando uma amplificação do olhar clínico e reconhecimento precoce das pessoas com maiores riscos de desenvolver novas LPP ou probabilidade de piora das mesmas. Tendo em vista que as formas de prevenção são de responsabilidade de toda a equipe interdisciplinar e buscando favorecer a ampliação da identificação precoce do risco de desenvolver LPP, também objetivou testar a possibilidade de predição do risco de desenvolver LPP, através da aplicação da escala de MIF em relação a escala de Braden.

\section{MÉTODO}

Trata-se de um estudo transversal, descritivo, com abordagem quantitativa, realizado no Centro Estadual de Reabilitação Dr. Henrique Santillo (CRER) no município de Goiânia. Foram entrevistados um total de 54 pacientes maiores de 18 anos, que tinham ao menos uma Lesão por Pressão e que estavam em tratamento no período do estudo. $O$ modo de seleção dos participantes seguiu conforme amostragem não probabilística, do tipo por acessibilidade e/ou conveniência.

A coleta de dados foi realizada através de entrevista, usando um questionário semiestruturado, elaborado pelos pesquisadores. A primeira e segunda parte do questionário englobou variáveis independentes sócio demográficas. A terceira parte abrangeu variáveis dependentes como: 
presença de morbidades de Hipertensão Arterial Sistêmica (HAS) e Diabetes Mellitus (DM), diagnóstico médico, tempo de lesão neurológica, capacidade funcional de acordo com a escala de MIF; dados antropométricos como altura e peso; risco de desenvolver LPP por meio da escala de Braden, quantidade e tempo de LPP, além do uso de superfícies de apoio para distribuição do peso corporal e suplemento nutricional, sendo os dados complementados com análise em prontuário eletrônico, quando necessário.

O período de coleta de dados foi de setembro a novembro de 2017, após aprovação do Comitê de Ética sob o parecer número 2.328.822 do Comitê de Ética em Pesquisa do Hospital Alberto Rassi (HGG). Após o término da coleta, os dados foram transcritos por um único pesquisador, em planilha Excel for Windows e analisados estatisticamente pelo software estatístico Statistical Package for Social Science (SPSS) versão 24.0. Primeiramente foi realizada uma análise descritiva das variáveis, sendo os resultados apresentados em tabelas contando frequência absoluta e percentual, alguns dados foram expressos em média e desvio padrão. Para correlação entre as variáveis tempo de lesão neurológica e estágio da LPP, foi aplicado o teste de Spearman. Com objetivo de testar a associação entre a escala de Braden e a escala de MIF, foi realizado um teste de regressão linear simples em conjunto com a correlação de Pearson, avaliando o valor preditivo entre as variáveis, considerando valor de $\mathrm{p}<0,05$ para que a amostra seja estatisticamente significativa.

Para construção da discussão teórica da temática em questão, optou-se por privilegiar periódicos de divulga- ção cientifica consultados na BVS, scielo e Pubmed utilizando os descritores em questão, priorizando periódicos publicados nos últimos dez anos.

\section{RESULTADOS}

Foram entrevistados ao todo 54 indivíduos que possuíam integridade da pele prejudicada e apresentavam pelo menos uma Lesão por Pressão. Os dados sócio demográficos encontrados são descritos na tabela $\mathrm{I}$.

Dos 54 participantes $46 \%$ residiam no interior do estado de Goiás, permanecendo no município de Goiânia somente para o tratamento de reabilitação. Quanto ao estado civil $44,4 \%$ eram solteiros, $24,1 \%$ eram casados, $14,8 \%$ eram viúvos, II, $1 \%$ se encontravam divorciados e $5,6 \%$ possuíam união estável.

Em relação a origem do recurso financeiro da família, $39 \%$ dos participantes eram aposentados, 39\% recebiam auxílio doença até análise do serviço de previdência social e $22 \%$ estavam desempregados, ou seja, dependiam da ajuda de familiares e amigos.

Quanto as morbidades, a maioria sendo $61,1 \%$ negaram portar HAS e DM, $18,5 \%$ relataram ter as duas morbidades em conjunto, $14,8 \%$ eram hipertensos e $5,6 \%$ possuíam diabetes mellitus. $\mathrm{Na}$ amostra foi observado valores de IMC entre 33,5 e II,5 com uma média 23,0 (desvio padrão $\pm 4,45$ ). Ao classificar os dados de acordo com o peso, evidenciamos que $53,7 \%$ possuíam peso normal, $20,4 \%$ estavam pré-obesos, $13 \%$ estavam abaixo do peso, $7,4 \%$ eram obesos e $5,6 \%$ se encontravam em sobrepeso.

Em relação ao nível de dependência funcional de acor-

TABELA 1 - Distribuição dos dados sócio demográficos de usuários de saúde.

Centro Estadual de Reabilitação Dr. Henrique Santillo, Goiânia, Goiás, 2017 (N=54)

\begin{tabular}{l|c|c}
\hline Variáveis & $F$ & $\%$ \\
\hline Sexo & 38 & 29,4 \\
\hline Masculino & 16 & \\
\hline Feminino & & 9,3 \\
\hline Idade & 5 & 64,8 \\
\hline Adolescência (18-21) & 35 & 25,9 \\
\hline Adulto (22-64) & 14 & \\
\hline Idoso (>65) & & 48,1 \\
\hline Escolaridade & 26 & 27,8 \\
\hline Analfabeto/ensino fundamental incompleto & 15 & 14,8 \\
\hline Ensino Fundamental Completo & 8 & 9,3 \\
\hline Ensino Médio Completo & 5 & \\
\hline Ensino Superior Completo & & \\
\hline Classe econômica & 3 & 16,6 \\
\hline B3 & 9 & 16,7 \\
\hline C1 & 9 & 61,1 \\
\hline C2 & 33 & \\
\hline D / E (até um salário mínimo) & & \\
\hline Fon & & \\
\hline
\end{tabular}

Fonte: dados da pesquisa, 2017 
do com a escala de MIF, foram encontrados os seguintes resultados descritos na tabela 2 .

Dividindo a escala em MIF motora, encontramos uma pontuação média de 34,6 (desvio padrão $\pm 26,8$ ), sendo que $31,4 \%$ dos participantes apresentaram dependência motora completa. Para MIF cognitiva, evidenciamos valores médios de 24,3 (desvio padrão \pm I2,6), a maioria com 48 , I \% mostraram totalmente independentes para necessidades cognitivas.

Pela limitação da capacidade funcional 59,3\% residiam com cônjuge, quando tinham, ou com filhos e $35,2 \%$ ainda residiam com os pais, revelando um público de cuidadores jovens e idosos, restando 5,6\% que moravam sozinho.

A tabela 3 a seguir, apresenta dados referentes as lesões neurológicas, complementando informações sobre a dependência funcional do indivíduo vítima de sequelas motoras e sensoriais.

Dentre os 54 participantes do estudo, foram relatadas um total de IIO LPP, sendo os locais anatômicos e estágios das lesões descritos a seguir na tabela 4 .

Das 110 LPP, $28 \%$ foram adquiridas na instituição de reabilitação onde foi realizada a pesquisa, sendo que destes, $64 \%$ se encontravam em cuidados semi-intensivos. $O$ restante de LPP correspondente a $72 \%$, foram adquiridas antes do paciente iniciar tratamento no centro de reabilitação, sendo admitido com LPP prévia.

Em relação ao tempo que o indivíduo permanece em tratamento de LPP, observamos que $44,4 \%$ tratavam LPP há três meses, $39,6 \%$ há mais de um ano, $14,8 \%$ entre sete meses a um ano e $\mathrm{II}, \mathrm{I} \%$ de quatro a seis meses.

Podemos confirmar através da aplicação do teste de Spearman, que existe uma correlação positiva e moderada entre $\circ$ tempo de lesão neurológica e o estágio da LPP, $(\rho=0,462 ; p<0,05)$, ou seja, quanto maior tempo um indivíduo apresenta uma sequela neurológica, pior tende a ser o estágio de uma LPP existente, conforme tabela 5 .

Analisando o risco de desenvolver LPP através da escala de Braden, evidenciamos os seguintes resultados apontados na tabela 6 .

Dentre os entrevistados $61,1 \%$ relataram fazer uso do colchão piramidal em domicílio, $17,1 \%$ possuíam o colchão pneumático e $14,8 \%$ disseram não fazer uso de nenhuma superfície de apoio que permite a distribuição do peso corporal. Em relação ao uso de suplemento de proteína para auxiliar no processo de cicatrização, 81,5\% alegaram não fazer uso e não estavam em acompanhamento com nutricionista.

TABELA 2 - Distribuição dos dados referente a dependência funcional de acordo com a escala de MIF. Centro Estadual de Reabilitação Dr. Henrique Santillo, Goiânia, Goiás, 2017 (N=54)

\begin{tabular}{lll}
\hline Variáveis & $\mathrm{F}$ & $\%$ \\
\hline Alta dependência funcional & 23 & 43 \\
Moderada dependência funcional & 17 & 31 \\
Baixa dependência funcional & 14 & 26 \\
\hline
\end{tabular}

Fonte: dados da pesquisa, 2017

TABELA 3 - Distribuição dos dados relacionados ao diagnóstico de lesão neurológica de usuários de saúde. Centro Estadual de Reabilitação Dr. Henrique Santillo, Goiânia, Goiás, 2017 (N=54)

\begin{tabular}{l|c|c}
\hline Variáveis & $F$ & $\%$ \\
\hline Diagnóstico médico & 26 & 48,1 \\
\hline Traumatismo raquimedular & 20 & 37,0 \\
\hline Acidente Vascular Cerebral & 5 & 9,3 \\
\hline Traumatismo crânioencefálico & 2 & 3,7 \\
\hline Encefalopatia por hipóxia & 1 & 1,9 \\
\hline Mielomeningocele & & 24,1 \\
\hline Tempo de lesão neurológica & 13 & 14,8 \\
\hline até 3 meses & 8 & 14,8 \\
\hline de 4 meses a 6 meses & 8 & 46,3 \\
\hline de 7 meses a 1 ano & 25 & \\
\hline acima de 1 ano & & 37,0 \\
\hline Sequelas & 20 & 25,9 \\
\hline Tetraplegia & 14 & 14,8 \\
\hline Paraplegia & 8 & 13,0 \\
\hline Hemiplegia D & 7 & 9,3 \\
\hline Hemiplegia E & 5 & \\
\hline Tetraparesia & & \\
\hline
\end{tabular}

Fonte: dados da pesquisa, 2017 
TABELA 4 - Distribuição dos dados referente as características das lesões por pressão. Centro Estadual de Reabilitação Dr. Henrique Santillo, Goiânia, Goiás, 2017 (N=110)

\begin{tabular}{|c|c|c|}
\hline Variáveis & $\mathrm{F}$ & $\%$ \\
\hline \multicolumn{3}{|c|}{ Locais anatômicos das LPP } \\
\hline Sacral & 37 & 33,6 \\
\hline Trocânter & 26 & 23,6 \\
\hline Calcâneo & 17 & 15,5 \\
\hline Ísquio & 9 & 8,2 \\
\hline Maléolo & 5 & 4,5 \\
\hline Outros & 5 & 4,5 \\
\hline Occipital & 2 & 1,8 \\
\hline Cotovelo & 2 & 1,8 \\
\hline Escápula & 2 & 1,8 \\
\hline Interglúteo & 1 & 0,9 \\
\hline \multicolumn{3}{|l|}{ Estágios das LPP } \\
\hline Estágio 2 & 13 & 11,8 \\
\hline Estágio 3 & 36 & 32,7 \\
\hline Estágio 4 & 37 & 33,6 \\
\hline
\end{tabular}

Fonte: dados da pesquisa, 2017

TABELA 5 - Teste de Spearman - Correlação entre o tempo de lesão neurológica e estágio de lesão por pressão. Centro Estadual de Reabilitação Dr. Henrique Santillo, Goiânia, Goiás, 2017

\begin{tabular}{|c|c|c|c|c|}
\hline & & & $\begin{array}{l}\text { Tempo de lesão neurológica } \\
\text { (meses) }\end{array}$ & $\begin{array}{l}\text { Estágio da lesão por } \\
\text { pressão }\end{array}$ \\
\hline \multirow{6}{*}{$\begin{array}{l}\text { Rô de } \\
\text { Spearman }\end{array}$} & \multirow{3}{*}{$\begin{array}{c}\text { Tempo de lesão } \\
\text { neurológica (meses) }\end{array}$} & Coeficiente de Correlação & 1,000 &, $462^{* *}$ \\
\hline & & Seg. (bilateral) & . &, 000 \\
\hline & & $\mathrm{N}$ & 54 & 54 \\
\hline & \multirow{3}{*}{$\begin{array}{c}\text { Estágio da lesão por } \\
\text { pressão }\end{array}$} & Coeficiente de Correlação &, $462^{\star *}$ & 1,000 \\
\hline & & Seg. (bilateral) &, 000 & \\
\hline & & $\mathrm{N}$ & 54 & 54 \\
\hline
\end{tabular}

**. A correlação é significativa no nível 0,01 (bilateral)

Fonte: dados da pesquisa, 2017

TABELA 6 - Distribuição dos dados referente ao risco de desenvolver lesão por pressão de acordo com a escala de Braden. Centro Estadual de Reabilitação Dr. Henrique Santillo, Goiânia, Goiás, 2017 (N=54)

\begin{tabular}{lccc}
\hline Variáveis & F & $\%$ \\
\hline Risco altíssimo & 1 & 1,9 & \\
\hline Risco alto & 20 & 37 & \\
\hline Risco moderado & 16 & 29,6 & \\
\hline Risco baixo & 17 & 31,5 & \\
\hline
\end{tabular}

Fonte: dados da pesquisa, 2017

A correlação de Pearson mostrou uma relação positiva e moderada entre as escalas de MIF e Braden.A regressão linear simples confirmou a hipótese de que a escala da MIF é preditora da escala de Braden quanto ao grau de risco do indivíduo desenvolver LPP, justificando cerca de $32 \%$ da amostra, $\left[F(I, 52)=25,440, p<0,00 I ; R^{2}=0,329\right]$, com ausência de auto-correlação evidenciado pelo valor de Durbin -Watson de 2,094. Podemos chegar ao escore da escala de Braden aplicando o escore resultante da escala de MIF na seguinte fórmula: Braden $=1 \mathrm{I}, 219+0,039$. (escala de MIF).

\section{DISCUSSÃO}

A predominância do sexo masculino em idade adulto- jovem, também é evidente em estudos com vítimas de Traumatismo Raquimedular (TRM), justificado pela maior exposição aos fatores etiológicos que estão inseridos na rotina diária destes indivíduos, já a prevalência de idosos corrobora com estudos direcionados a vítimas de Acidente Vascular Cerebral (AVC), visando que doenças cardiovasculares estão intimamente relacionadas ao aumento da longevidade ${ }^{(2-6)}$.

Os níveis de escolaridade predominantes foram analfabetos e ensino fundamental incompleto totalizando $48,1 \%$ da amostra, quanto a classe econômica, a maioria com $6 \mathrm{I}, 1 \%$ se enquadravam na classe $D$ e $E$ com renda familiar de até um salário mínimo, conforme demonstra- 
do na tabela I. Enfatizamos que a baixa escolaridade em conjunto com a baixa renda familiar, podem prejudicar diretamente a qualidade do cuidado de pessoas com maior dependência funcional, pois exigem suporte assistencial em tempo integral, além de recursos materiais complexos para manutenção da saúde ${ }^{(7)}$.

Dentre os 54 participantes se agregarmos os indivíduos solteiros, viúvos e divorciados, resultam em quase $70 \%$ da amostra total, e sabendo que a carga horária de cuidados exigidos por uma pessoa pode ser calculada pelo seu nível de dependência, no estudo em questão foi evidenciada que $42,6 \%$ necessitavam de três a seis horas de cuidados diários, demonstrando a indispensável presença de um acompanhante/cuidador para garantir necessidades básicas ${ }^{(4)}$.

Segundo Pereira e colaboradores ${ }^{(8)}$, a necessidade de cuidado em tempo integral muitas vezes envolve poucos componentes familiares gerando acúmulo de papéis e expondo o cuidador a sobrecarga física e emocional, podendo comprometer a eficiência do cuidado, pois algumas ações podem passar desapercebidas ou ganhar menor relevância pelos cuidadores, como a mudança de decúbito a cada duas horas por exemplo, sendo que é uma das principais formas preventivas de LPP.

As lesões cutâneas especificamente as provocadas por pressão excessiva, apresentam maior prevalência em pessoas com mobilidade física prejudicada, visto que a prevalência dos diagnósticos de TRM e AVC encontrados na amostra pode ser explanada em decorrência do aumento da violência urbana, aumento da expectativa de vida e avanços na área da medicina, que prolonga sobrevida de pacientes com doenças graves ${ }^{(9)}$.

Sabe-se que lesões neurológicas podem provocar alterações motoras, sensitivas e/ou cognitivas, o público estudado apresentou tetraplegia e paraplegia como as principais sequelas neurológicas desenvolvidas, sendo $37,0 \%$ e $25,9 \%$ respectivamente. Resultado divergente a outro estudo transversal, realizado em Maceió com vítimas de TRM, onde houve prevalência da sequela de paraplegia com $41,4 \%{ }^{(9)}$. Revelando um maior comprometimento motor, visto que tetraplegia acomete tronco, membros inferiores e membros superiores, deixando o indivíduo com maior grau de prejuízo na mobilidade física e consequentemente mais susceptível a desenvolver LPP.

Dentre os 54 sujeitos abordados na pesquisa, foram registrados uma totalidade de 110 LPP, resultando uma média de duas lesões para cada pessoa (desvio padrão $\pm 1,57)$. Discutindo as características das LPP, as regiões anatômicas de maior prevalência foram região sacral $33,6 \%$, seguida pela região trocantérica $23,6 \%$ e calcâneo 15,5\%. Dentre a classificação dos estágios das lesões por pressão, tivemos a predominância dos estágios quatro com $33,6 \%$ e três com $32,7 \%$, evidenciando a alta complexidade das lesões descritas na tabela 3.

Uma revisão de literatura voltada para discussão sobre medidas preventivas e tratativas de Lesão por Pressão, também evidenciou prevalência de LPP nas regiões sacrais, trocantéricas e calcâneos, justificado por serem locais de proeminência óssea mais eminente e que permanecem diretamente em contato com a superfície de apoio em pacientes acamados ${ }^{(10)}$.

O uso de curativos como profilaxia de LPP tem sido algo muito estudado atualmente, Santamaria e colaboradores $^{(11)}$ nos mostra que a cobertura composta de espuma de silicone em multicamadas tem oferecido resultados positivos na prevenção destas lesões em pacientes críticos, através de uma pesquisa randômica, em um hospital australiano envolvendo 440 pacientes, evidenciou uma redução de $10 \%$ da incidência de LPP no grupo controle que recebeu cuidados casuais associado ao uso da cobertura de silicone nas regiões sacral e calcâneo, quando comparado ao grupo experimental que recebeu somente cuidados usuais de prevenção.

Ao discutir os estágios das LPP e comparando aos resultados descritos em um estudo realizado por Mota e colaboradores $^{(12)}$, na mesma instituição deste estudo entre o período de 201 I e 2012 sobre a qualidade de vida em portadores de LM com úlceras por pressão, observamos uma mudança da prevalência dos estágios evoluindo para tecidos mais complexos, com aumento dos estágios três e quatro, mostrando a importância do acompanhamento do perfil do público atendido, o que facilita o reconhecimento de novas necessidades, além de nortear a atualização dos protocolos institucionais.

Um estudo envolvendo oito centros de reabilitação holandeses revelou que estágios de LPP três e quatro são característicos de pacientes com lesão medular crônica e que já foram reinseridos na comunidade após período de reabilitação hospitalar, contribuindo ao nosso estudo, visto que temos participantes com até 32 anos de lesão neurológica ${ }^{(13)}$.

Aplicando um teste estatístico sobre a amostra presente, podemos confirmar com a correlação de Spearman a existência de uma correlação positiva e moderada entre o tempo de lesão neurológica e o estágio da LPP, $(\square=0,462 ; p<0,05)$, ou seja, quanto maior tempo um indivíduo apresenta uma sequela neurológica, pior tende a ser o estágio de uma LPP existente, conforme tabela 4.

Em relação ao tempo em que as pessoas permaneciam em tratamento da LPP, foi encontrado valores entre um mês a oito anos, sendo que $44,4 \%$ relataram estar em tratamento da LPP por três meses e $29,6 \%$ se encontravam na luta em busca da cicatrização da ferida há mais de um ano. Permanecer por tempo prolongado com uma LPP, viabiliza o crescimento de bactérias multirresisten- 
tes no leito da ferida, elevando o risco de desenvolver bacteremia, caracterizado um dos piores desfechos clínico, pois associado ao uso constante de antibióticos de amplo espectro, surge a necessidades de cuidados intensivos, podendo evoluir para óbito, além de gerar gastos dispendiosos na manutenção de pacientes críticos $^{(14)}$.

Das 110 lesões por pressão registradas dentre os participantes da presente pesquisa, houve uma prevalência de $28 \%$ de LPP adquiridas na instituição estudada, mesmo sendo a maioria dos indivíduos sob cuidados semi-intensivos, revelou um dado preocupante quando comparado a literatura americana, que evidenciou uma taxa de prevalência entre $6,2 \%$ e 13,5\% através de um estudo de coorte e transversal, analisando instituições de cuidados intensivos e de reabilitação por um período de dez anos ${ }^{(15)}$.

A prevalência de LPP após a admissão gera um grande impacto para as instalações de reabilitação, segundo dados bibliográficos podem provocar repercussão negativa direta ao paciente, que sofre menor ganho motor, aumento do tempo de permanência na instituição hospitalar e menor chance de retornar à comunidade com boa qualidade de vida e manutenção dos ganhos ${ }^{(9)}$.

Uma pesquisa espanhola objetivando avaliar o impacto da incidência e prevalência de LPP em uma unidade de reabilitação percebeu através de uma análise longitudinal realizada com $26 \mathrm{I}$ pacientes, um acréscimo de 22 dias de internação aos pacientes com LPP, quando comparados aos pacientes com integridade tissular preservada, além de observar uma menor tendência desses pacientes a alcançar a independência funcional e uma ascensão no risco de desenvolver outras lesões por pressão(1).

Em contrapartida, as outras $72 \%$ das lesões por pressão descritas neste estudo, eram prévias, ou seja, foram adquiridas antes da admissão no centro de reabilitação, muitas vezes em instituições de pronto atendimento e unidade de terapia intensiva. Pacientes admitidos na emergência e transferidos para UTI estão comumente mais expostos a fatores de risco que contribuem para o desenvolvimento de uma LPP, por vezes, até fechar diagnóstico clínico, permanecem em superfícies rígidas para garantir imobilização, intensificado pelo grau e duração que permanece em estado de hipotensão, além do uso de drogas vasoativas ${ }^{(I I)}$. Destacando que uma LPP prévia pode ser caracterizada como um preditor clínico fortemente associado ao desenvolvimento de novas LPP em vítimas de TRM, segundo estudo de coorte observacional, ao elencar fatores de riscos associados a ulcera por pressão na reabilitação em 159 pacientes com lesões medulares, devemos investir de forma persistente em ações preventivas ${ }^{(16)}$.

Ao considerar que a pressão não aliviada provoca compressão da região tissular e comprometimento do fluxo sanguíneo, levando a morte tecidual, Picham e colaboradores $^{(17)}$ realizam estudos com intuito de comprovar a efetividade da mudança de decúbito a cada duas horas ou descompressão por pelo menos 15 minutos, frente a prevenção de LPP, através da instalação de sensores em pacientes de UTI, e indicação via monitor do momento certo da mudança de decúbito, buscando aumento da adesão desses protocolos nessas unidades, visto que a taxa de adesão é menor que $50 \%$.

Sabe-se da relevância de um aporte de nutrientes adequado, na contribuição da prevenção e cicatrização de LPP. Com intuito de avaliar o estado nutricional em pacientes amputados associada a LPP, um estudo no ambulatório de reabilitação em Curitiba, evidenciou indivíduos com sobrepeso associado ao déficit de proteínas e micronutrientes como, vitaminas $\mathrm{A}, \mathrm{C}$ e E, cobre e zinco, que são importantes no processo de cicatrização ${ }^{(18)}$. No presente estudo não foi investigado déficits de micronutrientes, porém 53,7\% apresentavam peso normal, $20,4 \%$ eram pré-obesos e ambos grupos possuíam LPP.

Também houve uma expressiva quantidade de indivíduos que não faziam uso de suplemento industrializado sendo $81,5 \%$. Resultados supostamente justificado pela maioria da amostra não se encaixar em quadros de desnutrição, porém, "Anholt, e colaboradores, concluíram que suplementos industrializados podem suprir as deficiências de micronutrientes, mesmo em pacientes sem risco nutricional que apresentam úlceras por pressão, reduzindo consideravelmente o tempo de recuperação da lesão"(18).

Quanto ao risco de desenvolver LPP, de acordo com a escala de Braden, 3I,5\% dos participantes apresentavam risco baixo, os demais $68,5 \%$ se enquadravam nos riscos moderados, altos e altíssimos, considerando que indivíduos com riscos altos são cerca de 25 vezes mais propensos a desenvolver o evento adverso, quando comparados as pessoas com risco baixo ou moderado, e que uma LPP prévia é um forte fator predisponente para desenvolvimento de outras lesões, a escala de Braden não deve ser usada de forma exclusiva para detecção do risco ${ }^{(19)}$.

Mertens e colaboradores ${ }^{(20)}$ evidenciaram que níveis de dependência funcional contribuem diretamente no risco de desenvolver LPP, e que uma escala de dependência de cuidados comumente utilizada na Alemanha, conhecida como Care Dependency Scale (CDS), funcionou bem para detecção de riscos de ulceras por pressão, sendo os fatores mais preditivos a continência, mobilidade e higiene do paciente.

Ao comparar a escala de Braden com a escala de MIF por ser a mais utilizada na instituição em estudo, para avaliação da dependência funcional, evidenciamos uma correlação positiva e moderada entre as mesmas, permitindo entender 
que quanto maior o escore da escala de MIF, maior o escore da escala de Braden, ou seja, quanto mais independente o indivíduo for, menor risco terá de desenvolver LPP.

Ao aplicar o teste de regressão entre as escalas supracitadas, mostramos que é possível prever a escala de Braden através da aplicação da escala de MIF, a partir da fórmula descrita anteriormente nos resultados, permitindo a equipe interdisciplinar ampliar o diagnóstico precoce do risco de desenvolver LPP, visto que a escala de Braden é usada majoritariamente pela equipe de enfermagem. Ressaltando que o teste realizado em estudo explica apenas $32 \%$ dos resultados da amostra, mesmo sendo estatisticamente significante e mostrando que o grau de dependência explica o risco de desenvolver LPP.

\section{CONCLUSÕES}

O público estudado apresentou uma prevalência de indivíduos do sexo masculino, com idade adulto-jovem, seguido de idosos, com baixo nível de escolaridade e baixa renda familiar. Com predominância do diagnóstico de TRM, evoluindo para tetraplegia como uma das principais sequelas neurológicas. Houve prevalência de indivíduos com alta dependência funcional, evidenciando maior comprometimento das atividades de mobilidade no leito e transferências entre superfícies de apoio.
Evidenciamos que a escala de MIF é um preditor da escala de Braden, a partir da correlação positiva e moderada entre as mesmas, nos permitindo garantir uma melhor comunicação entre a equipe interdisciplinar, visto que a escala de Braden está intimamente relacionada a assistência de enfermagem, sendo muitas vezes desconhecida por outros profissionais da área da saúde.

Tendo em vista que as formas de prevenção são de responsabilidade de toda a equipe interdisciplinar, o estudo contribui efetivamente para qualidade da assistência ao paciente com alterações neurológicas, ressaltando que conhecer as características sociodemográficas do público atendido e que a identificação precoce do risco de desenvolver LPP, permite um melhor planejamento das intervenções preventivas e orientações direcionadas ao paciente e aos cuidadores.

Como limitação deste estudo, podemos apontar que o mesmo foi realizado em uma única instituição de saúde, não permitindo abranger os resultados para a sociedade como um todo, além de considerarmos um amostra pequena para o teste de regressão que delimitou a predição da escala de Braden pela escala de MIF, no entanto sugere-se que futuras pesquisas merecem ser realizadas, com amostras maiores, visando um coeficiente de correlação mais robusto. 


\section{REFERENCIAS}

I. Pérez JMC, Pollo MCA, Aguilera MV, Grantham SJ, Sánchez AR, Ribeiro ASF. Repercusión de las heridas crónicas en las unidades de rehabilitación funcional. Gerokomos. 2015; 26(3): 109-I14.

2. Jacomo AE, Garcia ACF. Análise dos acidentes motociclísticos no Centro de Reabilitação e Readaptação Dr. Henrique Santillo (CRER). Acta Fisiátr. 20 I I; 18(3):I24- 129.

3. Riberto M, Miyazaki MH, Jucá SSH, Lourenço C, Battistella LR. Independência funcional em pessoas com lesões encefálicas adquiridas sob reabilitação ambulatorial. Acta Fisiátr.2007; |4(2):87-94.

4. DiVita MA, Granger CV, Goldstein R, Niewczyk P, Freudenheim JL. Risk Factors for Development of New or Worsened Pressure Ulcers Among Patients in Inpatient Rehabilitation Facilities in the United States: Data From the Uniform Data System for Medical Rehabilitation.American Academy of Physical Medicine and Rehabilitation. 2015; 7(6):599-6I 2.

5. Rogenski NMB, Kurcgant P. Avaliação da concordância na aplicação da escala de braden interobservadores. Acta Paul Enferm. 20I2; 25(I):24-28.

6.Scalzo PL, Souza ES, Moreira AGO,Vieira DAF. Qualidade de vida em paciente com acidente vascular cerebral: clínica de fisioterapia Puc Minas Betim. Rev Neurocienc. 2010; 18(10):I39-I44.

7.Viana RAS, Zuffi FB, Barduchi OhI RI, Chavaglia SRR. Perfil Socioepidemiológico de clientes com limitação de mobilidade e seus cuidadores. Rev. Enferm. UERJ. 20 I 3; 2 I (4):439-445.

8. Pereira RA, Santos EB, Fhon JRS, Marques S, Rodrigues RAP. Sobrecarga dos cuidadores de idosos com acidente vascular cerebral. Rev Esc Enferm USP. 2013; 47(I): I85-192.

9. Costa RC, Caliri MHL, Costa LS, Gamba MA. Fatores associados a ulcera por pressão em lesados medulares. Rev Neurocienc. 2013; 21 ( I):60-68.

10. Luz SR, Lopacinski AC, Fraga R, Urban CA. Úlceras de pressão. Geriatria \& Gerontologia. 2010; 4(I):36-43.

I I. Santamaria N, Gerdtz M, Sage S, McCann J, Freeman A,Vassiliou T,Vincentis S, Ng AW, Manias E.A randomised controlled trial of the effectiveness of soft silicone multi-layered foam dressings in the prevention of sacral and heel pressure ulcers in trauma and critically ill patients: the border trial. International Wound Journal. 201 3; I2(3):302-8.

12. Mota D, Ribeiro R, Barbosa M. Qualidade de vida em portadores de lesão medular com úlcera por pressão. Enfermería Global. 2016; I5(2): I3-39.

13. Verschueren JHM, Post MWM, Groot S, Woude LHV, Asbeck FWA, Rol M. Occurrence and predictors of pressure ulcers during primary in-patient spinal cord injury rehabilitation. Spinal Cord. 20 I I; 49(I): I06-I I 2.

14. Braga IA, Pirett CCNS, Ribas RM, Gontijo Filho PP, Diogo FiIho A. Bacterial colonization of pressure ulcers: assessment of risk for bloodstream infection and impact on patient outcomes. Journal of Hospital Infection. 20I3; 83(4):3।4-20.

15. VanGilder C, Lachenbruch C, Algrim-Boyle C, Meyer S. The International Pressure Ulcer PrevalenceTM Survey: 20062015.JWound Ostomy Continence Nurs. 2017;44(I):20-28.

16. Dejong G, Hsieh CJ, Brown P, Smout RJ, Horn SD, Ballard P, Bouchard T. Factors Associated with Pressure Ulcer Risk in Spinal Cord Injury Rehabilitation. American Journal of Physical Medicine \& Rehabilitation. 20 14; 93(I I):97I-86.

17. Pickham D, Ballew B, Ebong K, Shinn J, Lough ME,Mayer B. Evaluating optimal patient-turning procedures for reducing hospital-acquired pressure ulcers (LS-HAPU): study protocol for a randomized controlled trial.Trials. 2016;6(I): I7-190.

18. Teixeira ES, Pinto JD, Araújo CG, Baptista DR, Lopes JP.Avaliação do estado nutricional e do consumo alimentar de pacientes amputados e com úlceras de pressão atendidos em um Centro Hospitalar de Reabilitação. $O$ mundo da Saúde. 201 I; 35(4):448-453.

19. Fernandes LM, Silva L, Oliveira JLC, Souza VS, Nicola AL. Association between pressure injury prediction and biochemical markers. Rev Rene. 2016; I7(4):490-7.

20. Mertens RN, Halfens RJG, Dietz E, Ramona Scheufele BA, Theo Dassen RN. Pressure ulcer risk screening in hospitals and nursing homes with a general nursing assessment tool: evaluation of the care dependency scale. Journal of Evaluation in Clinical Practice. 2008; I4(6): 1018-25. 